\title{
Clinicopathological spectrum of small bowel obstruction and management outcomes in adults - experience at a regional academic hospital complex
}

\author{
MR Mthethwa, ${ }^{1}$ (D) C Aldous, ${ }^{2}$ (iD) TE Madiba ${ }^{3}$ (D) \\ ${ }^{1}$ Department of Surgery, Nelson R Mandela School of Medicine, Pietermaritzburg Hospitals Complex, University of KwaZulu-Natal, \\ South Africa \\ ${ }^{2}$ School of Health Science, Nelson R Mandela School of Medicine, University of KwaZulu-Natal, South Africa \\ ${ }^{3}$ Department of Surgery, Colorectal Unit, Nelson R Mandela School of Medicine, University of KwaZulu-Natal, South Africa
}

Corresponding author, email: musamthethwa70@gmail.com

Presented at the Meeting of the Surgical Research Society of Southern Africa in Bloemfontein, South Africa on 25-26 June 2015.

Background: Delay in operative management of small bowel obstruction (SBO) results in increased morbidity and mortality. The objective was to evaluate clinical presentation and treatment outcome of SBO.

Method: Prospective cohort study between 2013-2014. Adult patients presenting with SBO were included. Demographics, clinical details, investigations, operative findings, in-hospital progress and outcomes were documented.

Results: There were 156 patients (median age 37 [IQR 27-54 years]) with early (44) and delayed (112) presentation. M:F ratio was $1.4: 1$. Common causes of obstruction were adhesions $(94 ; 60.3 \%)$ and hernias $(31 ; 19.9 \%)$. Non-operative management was feasible in 59 patients $(37.3 \%)$ with a success rate of $71.2 \%$. Nonviable bowel was present in $45.1 \%$ (early $31 \%$, delayed $50 \% ; p=0.078)$. Sixty-one patients $(54 \%)$ underwent bowel resection; nine patients $(20.5 \%)$ in the early presentation group and $52(46.4 \%)$ in the delayed group $(p=0.003)$. Thirty-one patients needed ICU admission (early 5, delayed 26; $p=0.091)$. The delayed group had longer ICU stay $(p=0.018)$ and longer hospital stay $(p<0.001)$. There were more complications $(p=0.084)$ and re-laparotomies $(p=0.156)$ in the delayed group. Eight patients died $(5.1 \%)$.

Conclusion: The main causes of SBO were adhesions and hernias. Late presentation was associated with higher resection rate, higher critical care admission and longer hospital stay.

Keywords: small bowel obstruction, adhesive bowel obstruction, acute abdomen

\section{Introduction}

The diagnosis of intestinal obstruction is based on clinical and radiological evaluation and can be categorised as complete or partial. ${ }^{1-3}$ The former requires operative management and the latter should initially be managed non-operatively, especially when the most probable causes are adhesions or abdominal tuberculosis. ${ }^{1,3-5}$ Provided that the patient does not have peritonitis, the fluid shifts and electrolyte derangements caused by the proximal bowel dilatation can be corrected, and spontaneous resolution of the obstruction monitored clinically. ${ }^{4,6,7}$ These principles apply to both high-income countries (HICs) and low- and middle-income countries (LMICs). ${ }^{8-10}$ There is variation in the aetiology of small bowel obstruction (SBO) with adhesions from previous operations being a predominant aetiology in HICs, and hernias and tuberculosis remaining as the main causes in LMICs and countries with poorly resourced healthcare systems. ${ }^{9-13}$ Delay in reaching health care facilities is a variable that affects the system's ability to provide surgical intervention and is most marked in the poorly resourced health care settings..$^{7,13-15}$ Late presentation and undue delay in operative intervention for SBO may result in bowel ischaemia and increased need for resection with the resultant risk of complications. ${ }^{4,16,17}$ As an upper middle-income country with many rural communities, ${ }^{18}$ South Africa is well placed to investigate SBO and establish if the global trends seen in the international literature are evident in our setting. We, therefore, decided to undertake a study on SBO in our catchment area; the aim was to evaluate the aetiology and clinical patterns of SBO, reasons and patterns of delay to definitive surgical management, and the impact of delay on outcomes in our region.

\section{Methods}

The study was conducted at the Pietermaritzburg Academic Hospitals, namely Edendale and Grey's hospitals. Pietermaritzburg is the capital city of the KwaZulu-Natal (KZN) province, The two hospitals provide a full 24hour surgical service and serve the local community of the Pietermaritzburg metropole, as well as various district 
hospitals within the western region of the KZN province, which comprises a population of 3.5 million people. ${ }^{19}$

A prospective, cohort, observational study was conducted between 1 August 2013 and 31 July 2014. Patients older than 12 years, with an admission diagnosis or operative findings of SBO were identified. Those misdiagnosed, and those with colonic or functional SBO were excluded. Data captured included demographics, clinical presentation, physical findings, investigation results, aetiology of obstruction and management. Late presentation was defined as symptoms for more than two days prior to presentation. Patients were categorised into two groups, namely those receiving operative management (OM) group and those receiving nonoperative management (NOM) group. Patients in the NOM group were managed by keeping the patient nil by mouth, inserting a nasogastric tube (NGT) for decompression, intravenous fluid, electrolyte correction and close clinical monitoring. Patients suitable for NOM were given $100 \mathrm{ml}$ Gastrografin orally on admission or during the first 24 hours of admission to document bowel patency using abdominal radiographs.

The success of NOM was documented at the first passage of stools. Failure of NOM was recorded when an indication for operative intervention was identified by persistent symptoms (persistent vomiting, high NGT output, persistent abdominal pain and tenderness), clinical deterioration (tachycardia, worsening abdominal pain and tenderness) or confirmation of complete SBO on a Gastrografin radiographic study or on abdominal CT scan. $\mathrm{OM}$ was offered to patients with complete SBO, those with peritonism and severe SIRS with metabolic acidosis, and for failed NOM. Operative findings were recorded, including the obstructing pathology and the presence or absence of a nonviable bowel segment. Operative intervention and mode of abdominal closure were also recorded. Postoperative complications, postoperative critical care admission, and relaparotomy rate were recorded, as were the lengths of ICU and hospital stay (LOS). Follow-up was until the patient's discharge from hospital or death.

\section{Statistics}

Descriptive statistics were applied to the data. The chisquared test was used for categorical data and the independent $t$-test for continuous variables. Continuous data for hospital and ICU stay were expressed as mean and standard error of the mean (Mean [SEM]); the rest of the continuous data were expressed as the median and interquartile range

\begin{tabular}{lcc}
\multicolumn{3}{l}{ Table I: Aetiology of small bowel obstruction in $\mathbf{1 5 6}$ patients } \\
\hline Adhesive group (94 [60.3\%]) & \multicolumn{1}{l}{} \\
\hline Prior operations & 40 & Per cent \\
\hline Trauma laparotomy & 26 & 25.6 \\
Appendicectomy & 15 & 16.7 \\
Obstetrics/gynaecology & 13 & 9.6 \\
Gastrointestinal & & 8.3 \\
Non-adhesive group (62 [39.7\%]) & $\boldsymbol{n}$ & Per cent \\
\hline Pathology & 12 & 7.7 \\
\hline Inguinal hernia & 9 & 5.8 \\
Incisional hernia & 8 & 5.1 \\
Umbilical hernia & 2 & 1.3 \\
Epigastric hernia & 14 & 9 \\
Tumour/stricture & & \\
TB abdomen & 8 & 5.2 \\
Inflammatory mass & & 3.8 \\
Ileo-sigmoid knot & 6 & 1.3 \\
Small bowel volvulus & 2 & 0.6 \\
\hline
\end{tabular}

${ }^{1}$ Primary tumour (7), secondary tumour (7)

${ }^{2}$ Appendix mass (3), tubo-ovarian mass (3)

(IQR). A $p$-value of $<0.05$ was considered statistically significant.

\section{Results}

One hundred and fifty-six patients were enrolled in the study of whom 91 (58\%) were male (M:F ratio 1.4:1). The median age was 37 (IQR 27-54) years. There were 125 referrals: 94 $(60 \%)$ from district hospitals, $22(14 \%)$ from clinics, five (3\%) from other hospital departments and four $(3 \%)$ by local general practitioners. Thirty-one patients $(20 \%)$ were selfreferred from within the Pietermaritzburg metropole.

The main cause of SBO was adhesions from previous operations in 94 patients $(60.3 \%)$, followed by hernias in 31 patients $(19.9 \%)$. Other causes are detailed in Table I. Forty-four patients presented early and 112 were delayed. Of the 112 delayed presenters, 103 patients $(66 \%)$ presented late to our hospital, and a further nine were delayed within the hospital (6\%). The median delay was 4 (IQR 2-6) days. Reasons for the delay are shown in Table II. The most common reasons were inappropriate health-seeking behaviour $(60 ; 53.6 \%)$ and delayed referral $(52 ; 46.4 \%)$. District hospitals were the major point of delay, contributing

Table II: Patterns of delay in surgical management among 112 patients with small bowel obstruction

\begin{tabular}{llcc}
\hline Behavioural pattern & Reason for delay* & $\boldsymbol{n}$ & Per cent \\
\hline Delayed first presentation & Did not seek medical help & 22 & 19.6 \\
$n=50(44.6 \%)$ & Used alternate/herbal medicine & 15 & 13.4 \\
& Self-medication (OTC) & 13 & 11.6 \\
Delayed referral & Base hospital & 28 & 25.0 \\
$n=62(55.4 \%)$ & Clinic & 20 & 17.9 \\
& General practitioner & 5 & 4.5 \\
& Definitive hospital ${ }^{1}$ & 9 & 8.0
\end{tabular}

OTC - over-the-counter medicine

*Delay - Some patients had more than one point of delay: delay in base + GP practice (3); delay in base + clinic (3); delay in GP practice + clinic (1); delay in base + definitive hospital (2)

${ }^{1}$ Reason for delay at definitive hospital - Incorrect diagnosis/management (5); admitted to wrong specialty (4) 


\begin{tabular}{|c|c|c|c|c|c|}
\hline Clavien-Dindo classification & Complication & $\begin{array}{c}\text { Total } \\
n=77^{*}\end{array}$ & $\begin{array}{c}\text { Early } \\
n=16^{*}\end{array}$ & $\begin{array}{c}\text { Delay } \\
n=61 *\end{array}$ & $p$-value \\
\hline \multirow[t]{7}{*}{ Grade I } & Surgical site infection & 6 & 2 & 4 & \\
\hline & Prolonged ileus & 20 & 4 & 16 & \\
\hline & Renal dysfunction & 4 & 1 & 3 & \\
\hline & Hypocalcaemia & 1 & 1 & 0 & \\
\hline & Thrombocytopenia & 1 & 0 & 1 & \\
\hline & Hypoglycaemia & 1 & 0 & 1 & \\
\hline & Sub-total & $33(43 \%)$ & $8(50 \%)$ & $25(41 \%)$ & 0.520 \\
\hline \multirow[t]{4}{*}{ Grade II } & High output ileostomy & 1 & 0 & 1 & \\
\hline & Pneumonia & 7 & 2 & 5 & \\
\hline & Catheter-related sepsis & 2 & 0 & 2 & \\
\hline & Sub-total & $10(13 \%)$ & $2(12.5 \%)$ & $8(13 \%)$ & 0.958 \\
\hline \multirow[t]{4}{*}{ Grade III } & Enterocutaneous fistula & 1 & 0 & 1 & \\
\hline & Ischaemic bowel & 4 & 0 & 4 & \\
\hline & Peritonitis & 2 & 0 & 2 & \\
\hline & Sub-total & $7(9 \%)$ & $\mathbf{0}$ & $7(12 \%)$ & 0.148 \\
\hline \multirow[t]{8}{*}{ Grade IV } & Anastomotic failure & 5 & 1 & 4 & \\
\hline & Enterocutaneous fistula & 1 & 0 & 1 & \\
\hline & ARDS & 3 & 2 & 1 & \\
\hline & Septic shock & 3 & 0 & 3 & \\
\hline & MODS & 2 & 0 & 2 & \\
\hline & MI & 1 & 0 & 1 & \\
\hline & Pneumonia & 2 & 2 & 0 & \\
\hline & Sub-total & $17(22 \%)$ & $5(31.3 \%)$ & $12(20 \%)$ & 0.337 \\
\hline \multirow[t]{8}{*}{ Grade V } & Renal dysfunction & 2 & 0 & 2 & \\
\hline & Pneumonia & 1 & 0 & 1 & \\
\hline & Peritonitis & 1 & 0 & 1 & \\
\hline & Ischaemic bowel & 3 & 0 & 3 & \\
\hline & Pulmonary embolism & 1 & 0 & 1 & \\
\hline & MI & 1 & 1 & 0 & \\
\hline & MODS & 1 & 0 & 1 & \\
\hline & Sub-total & $10(13 \%)$ & $1(6.3 \%)$ & $9(15 \%)$ & 0.363 \\
\hline
\end{tabular}

*Some patients had more than one complication; ARDS - acute respiratory distress syndrome, MODS - multi-organ dysfunction syndrome, MI - myocardial infarction

to delay in 28 patients. Some of the late presenting patients had more than one point of delay.

Clinical presentations varied, with typical symptomatology of SBO observed in 135 patients $(86.5 \%)$. Complete obstruction was observed in 94 patients $(60.3 \%)$. Other positive findings were tachycardia $68(43.6 \%)$, fever 38 (24.4\%), leucocytosis 80 (51.3\%), peritonism 38 (24.4\%), and persistent tenderness $19(12.2 \%)$. All patients underwent plain abdominal X-ray. Sixty patients $(38.5 \%)$ were given oral Gastrografin with serial follow-through radiographs of whom six $(3.8 \%)$ needed an abdominal CT scan to finalise management decision. Fourteen $(9.0 \%)$ and four $(2.5 \%)$ patients respectively underwent only abdominal CT scan and ultrasound scan. Blood gas assessment showed normal blood gas $(54 ; 34.6 \%)$, metabolic alkalosis $(78: 50 \%)$, and metabolic acidosis $(24 ; 21.8 \%)$. Electrolytes were normal in 73 patients $(46.8 \%)$, abnormal in $55(35.3 \%)$ and 58 patients $(37.2 \%)$ had renal impairment.
Fifty-nine patients (37.8\%) were clinically suitable for NOM, of whom 22 and 37 were early and late presenters respectively. The median duration of NOM was 2 (IQR 2-4) days. NOM was successful in 42 of 59 patients $(71.2 \%)$ and the other 17 failed NOM and required operative intervention. Thus, 114 patients were considered for surgical intervention, but one patient refused intervention and was excluded from the analysis and discharged back to their base hospital. As a consequence, 155 patients were available for analysis (early 44, delayed 111).

One hundred and thirteen patients were offered operative intervention: 96 patients $(85 \%)$ at the outset and a further 17 patients $(15 \%)$ who failed NOM. Of the 17 patients who failed NOM, seven patients (41\%) needed bowel resection. Adhesiolysis was the most common intervention at laparotomy, carried out in 73 of 113 patients (64.6\%). Adhesions were also observed in other obstructions due to hernias, tuberculosis (TB) abdomen or inflammatory mass 


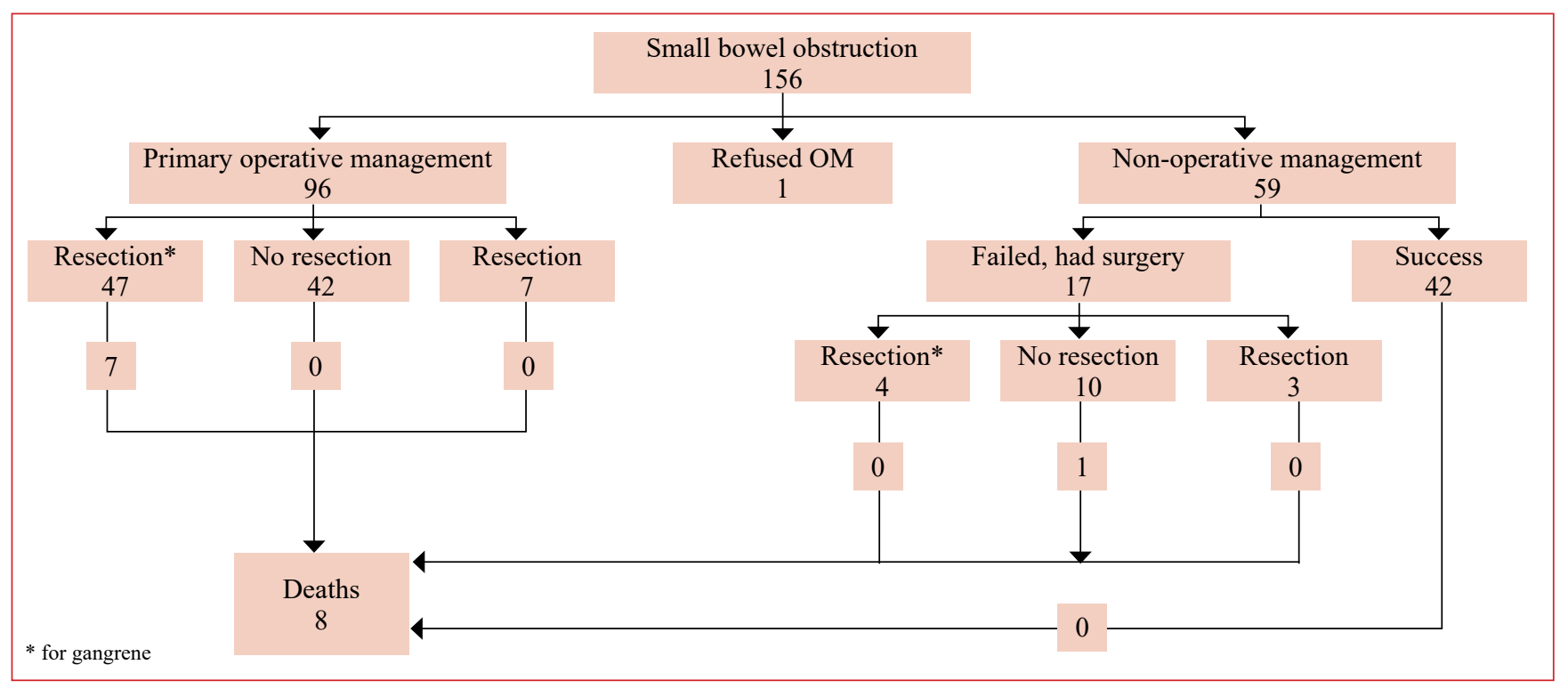

Figure 1: Flowchart of outcomes of small bowel obstruction observed during the study

(such as appendicular and tubo-ovarian complex); however, they were not the primary cause of SBO.

Figure 1 shows different pathways and outcomes of patients presenting with SBO during this study. Nonviable bowel was seen in 51 of 113 patients (45\%) undergoing laparotomy; nine $(31 \%)$ from the early presentation group and $42(50 \%)$ from the delayed group $(p=0.078)$. Bowel resection was undertaken in 61 of 113 patients $(53.9 \%)$, including the seven of the 17 patients who failed NOM. Thus nine patients $(20.5 \%)$ in the early presentation group and $52(46.4 \%)$ in the delayed group underwent resection $(p=0.003)$. Other interventions were hernia repair (25) and appendicectomy (3). A stoma was deemed necessary in 12 patients undergoing bowel resection. Appendicectomy was performed in SBO due to appendix inflammatory mass. Fifteen patients required temporary abdominal closure (TAC) using Bogota bag.
Thirty-one patients required critical care admission (27.4\%); five in the early group (11.4\%) and $26(23.4 \%)$ in the delayed group $(p=0.091)$. Three patients $(7 \%)$ required relaparotomy in the early group as opposed to $20(18 \%)$ in the delayed group $(p=0.156)$.

Fifty-five patients $(35.3 \%)$ developed complications; 11 of 44 in the early group (25\%) and 44 of 111 in the delayed group (37\%). The 55 patients developed 77 complications (44 intra-abdominal and 33 extra-abdominal complications). Table III depicts different complications, according to the Clavien-Dindo classification system. ${ }^{20}$ Eleven out of 29 patients with early presentation (37.9\%) developed 16 complications and 44 patients out of 84 patients with delayed presentation (52\%) developed 61 complications $(p=0.084)$. The delayed group had more complex complication patterns. Seventeen patients had two complications each, and seven had three each. In addition, from the delayed presentation group, 12 and eight patients had an un-reversed stoma and

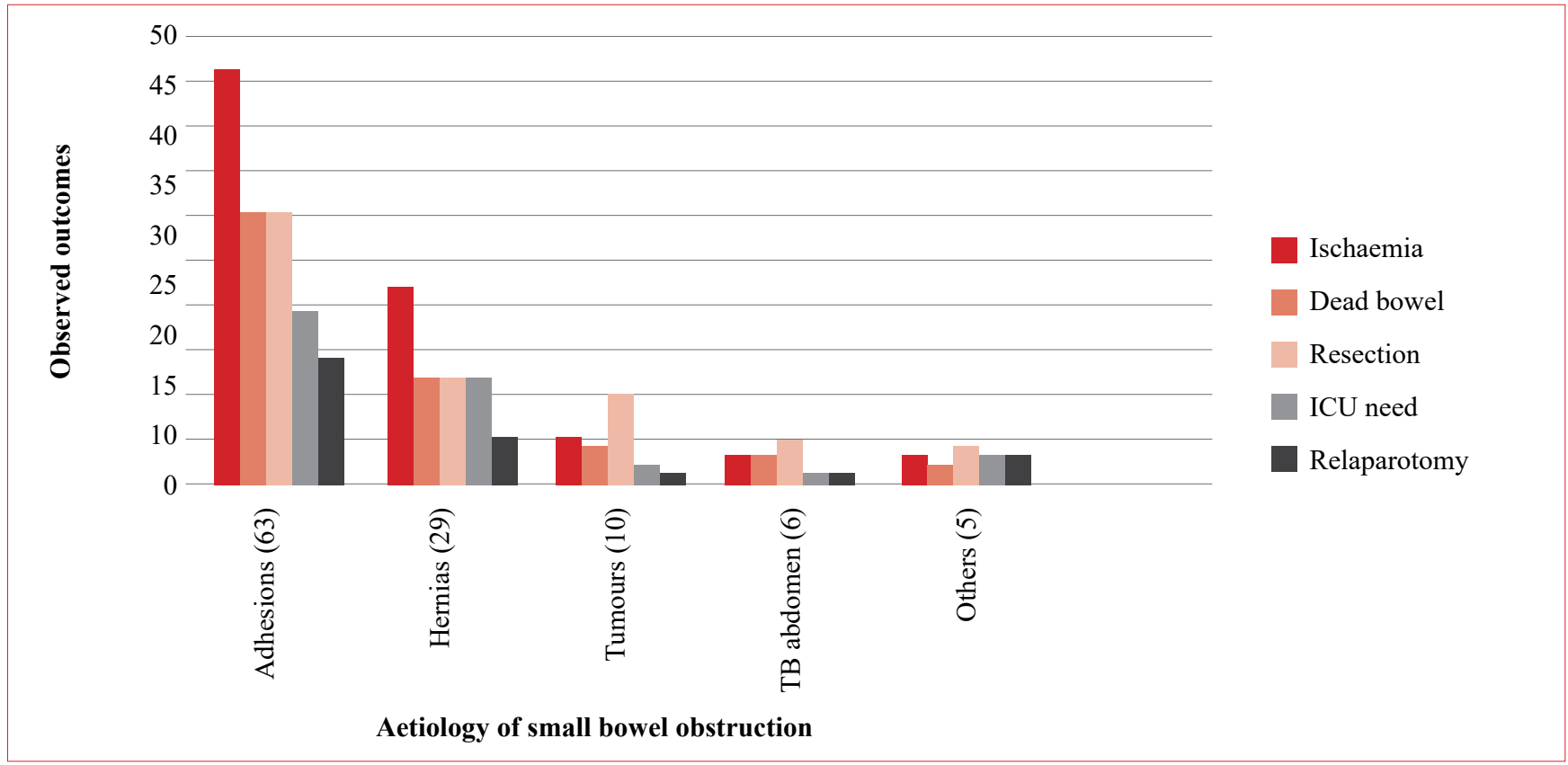

Figure 2: The graph shows the degree of bowel viability, need for critical care and reoperation related to aetiology 
an open abdomen, respectively. Eight patients (5.1\%) died, seven $(6.3 \%)$ from the delayed group and one $(2.3 \%)$ from the early presentation group $(p=0.442)$.

The causes of mortality were myocardial infarction (MI) (1), pulmonary embolism (1), multiple organ dysfunction (5), for the delayed group, and MI (1) for the early presentation group.

Figure 2 demonstrates the different aetiologies together with associated complications. Adhesions and hernias were the most common causes of obstruction and thus contributed to most of the complications.

The mean hospital stay was 8.7 (SEM 0.6) days; the mean hospital stay for the early group was 5.6 (SEM 0.6) days vs 9.9 (SEM 0.8) days for the delayed groups $(p<0.001)$. The mean ICU stay was 1.5 (SEM 0.4) days; the mean ICU stay for the early group was 0.5 (SEM 0.2) days as opposed to 1.9 (SEM 0.5) days for the delayed group $(p=0.018)$.

\section{Discussion}

The aetiology and pattern of SBO have been the topics of extensive research in various settings. It differs in LMICs which are poorly resourced from that in well-resourced HICs. ${ }^{1,3,5,11,12}$ Hernias are the leading cause of SBO in LMICs, whereas postoperative adhesions are the main cause in the HICs. ${ }^{10-13}$ The most common cause in this series was adhesions from previous operations, much as in the HICs. However, the common operations leading to adhesions were laparotomies for trauma followed by appendicectomy as opposed to other parts of the world where appendicectomy and colorectal operations are the main index operations leading to subsequent development of adhesive SBO. ${ }^{11}$ This is probably related to a higher incidence of trauma-related laparotomy in our setting. ${ }^{21}$ All types of hernias, namely congenital and acquired, were the second major cause at $19.9 \%$ which is higher than $10 \%$ in $\mathrm{HICs}^{1,5,12}$ and lower than $36 \%$ reported from other LMICs. ${ }^{11-13}$

Surgical candidates in this series had a variable clinical presentation. The majority of patients presented late, and there were different points of delay. Patient factors included lack of insight, thinking the abdominal symptoms would resolve in time, attempting self-medication or herbal medication. Factors related to the primary health caregivers included incorrect diagnosis, inappropriate management at primary health care levels, and multilevel consultations. Delay at the base hospital was the most frequent point of delay. Delays at the definitive hospitals were related to misdiagnosis and admission to the wrong speciality and further diagnostic imaging. In a study by Malangoni et al. of 366 patients, 222 were admitted to surgical service and 114 to medical service, operated patients admitted to surgical service had shorter preoperative (2.7 vs 6.3) days, $p<0.01$ and overall LOS (17.9 vs 22.8) days, $p<0.0001 .^{22}$ Multilevel consultation is another factor that increases the delay to definitive hospital and surgical intervention. In an emergency departmentbased study, of 355 advised admissions, 238 (67\%) were delayed. The common reason for delay was multiple consultations with further investigations, $p<0.001$ and file making process, $p<0.001 .^{23}$ Incorrect diagnosis results in patients being sent back home and then re-consulting to the same point or elsewhere a day or so later, before they re-join the referral ladder. There is evidence to suggest that race may play a role in influencing delay in presentation for medical attention. A North American outcome comparison study among different races discovered that Black patients had relatively longer delays irrespective of access to health care facility. ${ }^{8}$ Inter-hospital transfer was another cause of delay in this series. An Australian study by Limmer et al. demonstrated that inter-hospital transfer tends to delay surgical intervention and increases the length of hospital stay. Amongst the 910 adults patients in the study who underwent emergency abdominal surgery, 290 (31.9\%) were transferred in from local district hospitals, had delayed surgical intervention $(14.2 \mathrm{~h}, p=0.001)$, postoperative LOS 1.1 days $(p=0.001)$ and overall LOS 1.6 days $(p<0.001)$ - observed in all emergencies except peptic ulcer perforations. ${ }^{24}$ Other reasons for delay include the time since the onset of illness because of late presentation due to lack of health care facilities, lack of health awareness, ignorance and poverty. ${ }^{13,15}$ Late presentation and undue delay in operative intervention for SBO results in bowel ischaemia, increases the need for resection and the risk of complications. $^{4,16,17}$

There was nonviable bowel in $45 \%$ of cases. Bowel resection was necessary in $54 \%$ of cases with the vast majority coming from the delayed group. Admittedly not all resections are necessarily due to late presentation but may be necessitated by the condition responsible for the obstruction, namely tumours or strictures.

Fifty-four per cent of patients underwent resection in this study, which is higher than the 36\% (range 9-51\%) reported in the literature. ${ }^{25,26}$ In a study by Meier et al., resection was necessary in $32.4 \%$ and there was a longer hospital stay for the OM group, and nine deaths (6.6\%) were observed, all from the OM group. ${ }^{26}$ The complication rate for the whole group was $35 \%$ (25\% and $37 \%$ for the early presentation and delayed presentation groups, respectively). These differences, however, did not reach statistical significance. A systematic review of nine studies by Thornblade et al. demonstrated an average mortality of $4 \%$ after surgery and $2 \%$ after NOM. ${ }^{25}$ The same review demonstrated a shorter hospital stay after NOM than after OM. ${ }^{25}$

This study demonstrates that delay in operative management of SBO is associated with a significantly increased rate of bowel resection, re-laparotomy rate and a longer LOS. There is also a trend that delay is also associated with higher ICU usage and longer ICU stay, and a high rate of complex morbidity and mortality. Other authors have confirmed that delay before intervention in SBO is associated with increased morbidity and mortality. ${ }^{8,10,16,25}$ Thornblade et al. ${ }^{25}$ reported lower resection rates in the early surgery group than in the delayed surgery group. SSI rates were similar and a longer average LOS for the delayed surgery group. ${ }^{25}$

NOM of SBO is acceptable in patients with a partial obstruction due to adhesions or abdominal tuberculosis in the absence of persistent tachycardia, fever, leucocytosis, metabolic acidosis, septic shock, persistent or progressive abdominal pain and tenderness, or peritonism..$^{1,3,5,25}$ This involves keeping the patient nil by mouth, nasogastric tube insertion where necessary, initiation of anti-TB treatment in cases of abdominal TB, ${ }^{9}$ and regular clinical and radiological evaluations for improvement or deterioration. ${ }^{27,28}$ Oral feeds are introduced when symptoms and signs abate. In this study, 59 patients were candidates for NOM. The success rate following NOM was $72.4 \%$, and there was no difference between early and delayed presenters. The role of NOM in patients with delayed presentation has not been described 
in the literature. The observed success rates in the delayed group are said to be related to obstructive morphology, namely matted adhesions versus adhesive band. ${ }^{3,29}$ The average duration of NOM for this study was 3.15 days in this series. Although evidence for the optimal duration of NOM is absent, most authors suggest that NOM should not extend beyond 3-5 days for non-resolving SBO, even in the absence of clinical deterioration. ${ }^{3,5,16}$

Current management guidelines support the use of watersoluble contrast meal and follow-through as an adjunct to NOM. ${ }^{3,5,30}$ This improves time to bowel function recovery and reduces overall hospital stay. ${ }^{30}$ The dose is 50 to 150 $\mathrm{ml}$ given orally or via nasogastric tube, on admission or at 48 hours of unsuccessful NOM. The likelihood of treatment success is demonstrated by the presence of contrast at the ileocaecal junction as visualised on an erect abdominal X-ray. ${ }^{30}$ Failure of progress is diagnostic of complete obstruction and is an indication for operative intervention. ${ }^{1,3,5}$ In the current study, a Gastrografin meal and follow-through was given in 60 patients, and it influenced decision-making in 56 patients. The earlier the Gastrografin meal and follow-through is given, the sooner the diagnosis of complete SBO is made, thus avoiding further delays. The use of Gastrografin in this study was limited to differentiating partial from complete SBO. It is crucial that the patient is adequately hydrated before this investigation is undertaken. ${ }^{30}$

Current management guidelines recommend abdominal CT scan for all patients with suspected SBO to confirm SBO and assess the risk of small bowel ischaemia., ${ }^{1,3,5}$ Furthermore, abdominal CT scan has a value in patients posing a diagnostic dilemma and those with a history of malignancy, prior irradiation or those with possible abdominal TB. ${ }^{1,3}$ Abdominal CT is, however, of limited availability in resource-challenged settings, and, in this study, it was necessary for 20 patients where it was useful in defining the presence, cause, the site and degree of SBO. Most notably, the turnaround time for this investigation was long, a common issue in resource-limited settings. In patients who present with a possibility of undiagnosed underlying malignancy, it is advisable to perform this investigation in patients who respond to NOM even on an elective basis. ${ }^{28,31}$

\section{Conclusion}

The leading cause of SBO in adult patients in our region is adhesions from previous operations followed by hernias. Most patients present late and/or are delayed by the referral process. Delayed presentation was associated with higher resection rate, higher critical care admission and prolonged hospital stay. Morbidity and mortality rates were higher in the delayed groups although the difference in morbidity and mortality did not reach statistical significance. While NOM is appropriate in selected cases, proper clinical evaluation and use of further imaging modalities may be invaluable in deciding on the need for surgery. We encourage active preventive measures, including early repair of hernias, improved education for patients at risk and heightened awareness at primary health care level to ensure early diagnosis and early referral to centres with appropriate surgical facilities.

\section{Acknowledgements}

Dr Yoshan Moodley, University of KwaZulu-Natal, for statistical analysis.

\section{Conflict of interest}

All authors declare no conflict of interest.

\section{Funding source}

No funding was required.

\section{Ethical approval}

Ethical approval was obtained from the Biomedical Research Ethics Committee (BREC) of the University of KwaZuluNatal (REF: BE207/13). Informed consent was obtained from each participant, and confidentiality was maintained by using study codes as identifiers instead of names.

\section{ORCID}

MR Mthethwa (D) https://orcid.org/0000-0002-5374-050X

C Aldous (iD https://orcid.org/0000-0002-7199-9160

TE Madiba (D) https://orcid.org/0000-0002-0155-9143

\section{REFERENCES}

1. Maung AA, Johnson DC, Piper GL, et al. Evaluation and management of small-bowel obstruction - an Eastern Association for the Surgery of Trauma practice management guideline. J Trauma Acute Care Surg. 2012;73:S362-9. https:// doi.org/10.1097/TA.0b013e31827019de.

2. Zielinski MD, Bannon MP. Current management of small bowel obstruction. Adv Surg. 2011;45:1-29. https://doi org/10.1016/j.yasu.2011.03.017.

3. Ten Broek RPG, Krielen P, Di Saverio S, et al. Bologna guidelines for diagnosis and management of adhesive small bowel obstruction (ASBO) - 2017 update of the evidencebased guidelines from the world society of emergency surgery ASBO working group. World J Emerg Surg. 2018;13:24 https://doi.org/10.1186/s13017-018-0185-2.

4. Aldemir M, Yağnur Y, Taçyildir I. The predictive factors for the necessity of operative treatment in adhesive small bowel obstruction cases. Acta Chir. Belg. 2004;104:76-80. https:// doi.org/10.1080/00015458.2003.11681150.

5. Rami Reddy SR, Cappell MS. A systematic review of the clinical presentation, diagnosis, and treatment of small bowel obstruction. Curr Gastroenterol Rep. 2017;19:28-42. https:// doi.org/10.1007/s11894-017-0566-9.

6. Kumari N, Charokar K, Bharang K. Study of clinical presentation and management of intestinal obstruction and its evaluation with respect to morbidity and mortality. Int J Surg Orth. 2020;6:166-72. https://doi.org/10.17511/ijoso.2020.i03. 05 .

7. Shih SC, Jeng KS, Lin SC, et al. Adhesive small bowel obstruction - how long can patients tolerate conservative treatment? W J Gastroenterol. 2003;9:603-5. https://doi. org/10.3748/wjg.v9.i3.603.

8. Chiu AS, Jean RA, Davis KA, Pei KY. Impact of race on the surgical management of adhesive small bowel obstruction. J Am Coll Surg. 2018;226:968-76. https://doi.org/10.1016/j. jamcollsurg.2017.11.006.

9. Chalya PL, Mchembe MD, Mshana SE, et al. Tuberculous bowel obstruction at a university teaching hospital in northwestern Tanzania - a surgical experience with 
118 cases. World J Emerg Surg. 2013;8:1-9. https://doi. org/10.1186/1749-7922-8-12.

10. Ohene-Yeboah M, Adippah E, Gyasi-Sarpong K. Acute intestinal obstruction in adults in Kumasi, Ghana. Ghana Med J. 2006;40:50-4.

11. Malik AM, Shah M, Pathan R, Sufi K. Pattern of acute intestinal obstruction - is there a change in the underlying etiology? Saudi J Gastroenterol. 2010;16(4):272-4. https:// doi.org/10.4103/1319-3767.70613.

12. Ullah S, Khan M, Mumtaz N, Naseer A. Intestinal obstruction - a spectrum of causes. JPMI. 2009:23(2):188-92.

13. Moalim A, Fiqi A, Dalmar A, Al E. Factors associated with intestinal obstructions among adults in Keysaney Hospital, Mogadishu, Somalia. Int J Hum Heal Sci. 2017;1:70-8.

14. Mbah N, Opara W, Agwu N. Waiting time among acute abdominal emergencies in a Nigerian teaching hospital - causes of delay and consequences. Niger J Surg Res. 2006;8:69-73.

15. Guzman I, Cuesta J, Trelles M, Al E. Delays in arrival and treatment in emergency departments: Women, children and non-trauma consultations the most at risk in humanitarian settings. PLoS One. 2019;14(3):e0213362. https://doi.org/1 0.1371/journal.pone.0213362.

16. CarmichaeL J, Mills S. Reoperation for small bowel obstruction - how critical is the timing? Clin Colon Rectal Surg. 2006;19:181-7. https://doi.org/10.1055/s-2006-956438.

17. Fevang BT, Fevang JM, Søreide O, Svanes K, Viste A. Delay in operative treatment among patients with small bowel obstruction. Scand J Surg. 2003;92:131-7. https://doi. org/10.1177/145749690309200204.

18. UNDP. United Nations Development Programme. Human Development Index (HDI). 2020.

19. Lehotla P. Provincial profile: KwaZulu-Natal Census 2011. 2011.

20. Dindo D, Demartines N, Clavien PA. Classification of surgical complications - a new proposal with evaluation in a cohort of 6336 patients and results of a survey. Ann Surg. 2004;240:20513. https://doi.org/10.1097/01.sla.0000133083.54934.ae.
21. Mnguni MN, Muckart DJJ, Madiba TE. Abdominal trauma in Durban, South Africa - factors influencing outcome. Int Surg. 2012;97(2):162-8. https://doi.org/10.9738/CC84.1.

22. Malangoni MA, Times ML, Kozik D, Merlino JI. Admitting service influences the outcomes of patients with small bowel obstruction. Surgery. 2001;130:706-13. https://doi. org/10.1067/msy.2001.116918.

23. Tashkandy MA, Gazzaz ZJ, Farooq MU, Dhafar KO. Reasons for delay in inpatient admission at an emergency department. J Ayub Med Coll Abbottabad. 2008;20:38-42.

24. Limmer AM, Edye MB. Interhospital transfer delays emergency abdominal surgery and prolongs stay. ANZ J Surg. 2017;87:867-72. https://doi.org/10.1111/ans.13824.

25. Thornblade LW, Verdial FC, Bartek MA, Flum DR, Davidson $\mathrm{GH}$. The safety of expectant management for adhesive small bowel obstruction - a systematic review. J Gastroint Surg. 2019;23:846-59. https://doi.org/10.1007/s11605-018-4017-1.

26. Meier RPH, Oulhaci De Saussure W, Orci LA, et al. Clinical outcome in acute small bowel obstruction after surgical or conservative management. World J Surg. 2014;38:3082-8. https://doi.org/10.1007/s00268-014-2733-6.

27. Paulson EK, Thompson WM. Review of small bowel obstruction - the diagnosis and when to worry. Radiology. 2015;275:332-42. https://doi.org/10.1148/radiol.15131519.

28. Maglinte D, Heitkamp D, Howard T, et al. Current concepts in imaging of small bowel obstruction. Radiol Clin N Am. 2003;41:263-83.

29. Bueno-Lledó J, Barber S, Vaqué J, et al. Adhesive small bowel obstruction: predictive factors of lack of response in conservative management with Gastrografin. Dig Surg. 2016;33:26-32. https://doi.org/10.1159/000441530.

30. Shingla R, Singh B, Kumar A, Al E. Role of Gastrografin contrast study in intestinal obstruction. Int $\mathrm{J}$ Anat Radiol Surg. 2017;6:S001-5. https://doi.org/10.7860/ IJARS/2017/28170:2285.

31. Hayanga AJ, Bass-Wilkins K, Bulkley GB. Current management of small-bowel obstruction. Adv Surg. 2005;39:1-33. https://doi.org/10.1016/j.yasu.2005.05.001. 